Plant Science 161 (2001) 635-644

\title{
Regulation of starch granule-bound starch synthase I gene expression by circadian clock and sucrose in the source tissue of sweet potato
}

\author{
Shu-Jen Wang, Kai-Wun Yeh, Chia-Yin Tsai * \\ Department of Botany, National Taiwan University, No. 1, Section 4, Roosevelt Road, Taipei, Taiwan, ROC
}

Received 12 February 2001; accepted 23 April 2001

\begin{abstract}
Studies on regulations of transitory starch synthesis and degradation in the leaf tissue are important for understanding how carbons could be distributed effectively from the source to sink tissues. Therefore, expressions of starch granule-bound starch synthase I (GBSSI) gene in leaves of sweet potato were studied under different photoperiodic conditions and various sugar treatments. Results indicated that accumulations of GBSSI mRNA and its protein were controlled by an endogenous biological clock. Starch accumulations in leaves also showed a pattern characteristic of circadian rhythm. In addition to circadian clock, sucrose also played an important role in regulating GBSSI mRNA accumulations. Although sucrose stimulated the transcription of GBSSI, it had no effect on the rhythmic pattern of GBSSI gene expressions. Protein phosphorylation/dephosphorylation were involved in the sucrose-related signal transduction for GBSSI gene expressions. However, the sugar sensing for regulation of GBSSI was independent of the hexokinase-mediated pathway. In conclusion, the GBSSI gene expression in leaves of sweet potato appears to be regulated by two independent pathways. First, light is responsible for setting up biological clock(s) that control the circadian expression of GBSSI gene; and second, light plays an indirect signal to enhance GBSSI mRNA accumulations mediated by the photosynthetic product, sucrose. (C) 2001 Elsevier Science Ireland Ltd. All rights reserved.
\end{abstract}

Keywords: Circadian rhythm; Granule-bound starch synthase I; Ipomoea batatas; Sink-source; Sucrose effect

\section{Introduction}

Starch granule-bound starch synthase I (GBSSI), also known as the WAXY protein, has been shown to be an important enzyme for the synthesis of amylose in the sink tissue [1-3]. However, expressions of this gene relative to starch metabolism in the source tissue remain unclear. We have isolated a GBSSI gene from sweet potato (Ipomoea batatas), and this gene was well expressed in tuberous roots, leaves, and stems. In tuberous roots, a sink tissue where accumulations of GBSSI mRNA increased along with developmental stages. On the other hand, mRNA levels in leaves fluctuated and exhibited a circadian rhythm during a 16-h light/8-h dark (16 L/8 D) photoperiod [4]. The circadian expres-

\footnotetext{
* Corresponding author. Tel.: + 886-2-2363-7964; fax: + 886-22391-8940.

E-mail address: tsaicy@ccms.ntu.edu.tw (C.-Y. Tsai).
}

sion of GBSSI gene was also observed in snapdragon [5].

Circadian rhythm is defined as a phenomenon controlled by endogenous $\operatorname{clock}(\mathrm{s})$, and the free-running period of the clock is about $24 \mathrm{~h} \mathrm{[6].} \mathrm{Light} \mathrm{is} \mathrm{considered}$ as an input signal that may set up circadian clocks, and the central clock regulates gene expressions through various signal transduction pathways [7]. Most of the circadian clock-controlled genes identified are involved in photosynthesis, carbon and nitrogen metabolisms. Periodic mRNA fluctuations of chlorophyll $\mathrm{a} / \mathrm{b}$ binding protein $(C a b)$ gene have been observed in various plant species $[8,9]$. In the area of carbon metabolism, expressions of ribulose-1,5-bisphosphate carboxylase/oxygenase $(R u B i s C O)$ gene have also been shown to exhibit circadian rhythms [10]. Nitrate and nitrite reductases are two major enzymes involved in the nitrogen metabolic pathway, and their expressions are controlled by biological clocks as well [11]. Furthermore, several 
genes that are not related to carbon and nitrogen metabolisms, e.g. catalase genes, were also observed to be under circadian controls [12].

In addition to the effect on gene expressions, endogenous circadian clocks have been shown to affect starch accumulation in several plant species. When sugar beet was grown under a 14-h photoperiod, accumulation of starch in leaves reached its maximum approximately 4 $\mathrm{h}$ after the beginning of illumination, and maintained steady until $2 \mathrm{~h}$ before the end of the light period. The pattern of starch accumulations was similar even though irradiance continued to sustain photosynthesis [13]. Although mechanisms controlling the circadian pattern of starch accumulation are still unclear, enzymes involved in the synthetic pathway were suggested as regulators [13]. A similar observation was made in Snapdragon [5].

While light may function as a signal to set up biological clocks, sugars also play an important role in controlling gene expressions related to photosynthesis and carbohydrate metabolism in plants [14]. For carbons metabolism, genes related to starch synthesis, such as ADP-glucose pyrophosphorylase, GBSSI and branching enzyme have been observed to be regulated by sugars [15-18]. Although sugar effects on gene regulations were observed in many plant systems, the definition of sugar signals is still not well established. Certain effects of sucrose could be substituted by glucose or fructose, suggesting that sucrose was not a direct signal [19]. However, sucrose could serve as a signal molecule to regulate gene expressions mediated by a specific sensor and signal transduction pathway [20].

Three different sugar-sensing systems have been postulated in plants [21,22]. First, hexokinase serves as an intracellular sensor in a way similar to that of the yeast system. Second, hexose-transporter functions as a sugar sensor located on plasma membrane. Third, sucrosetransporter plays a major role in sugar sensing. Several studies suggest that certain cascades of sugar-related signal transductions in higher plants might be similar to those of the yeast, and mediated by protein phosphorylation/dephosphorylation processes [22]. For example, protein phosphatase inhibitors strongly inhibited sucrose-inducible accumulations of the small subunit of ADP-glucose pyrophosphorylase mRNA in petioles of sweet potato [16].

Because sucrose is a major photosynthetic product and a precursor for starch synthesis, effects of light and sucrose on GBSSI gene expressions were studied in leaves of sweet potato. Furthermore, the accumulation patterns of GBSSI mRNA and protein were compared with the profile of starch accumulation. It is anticipated that these studies should provide information for a better understanding on regulatory mechanisms of $G B$ $S S I$ expression in the source tissue as well as carbon distribution from the source to sink tissues.

\section{Materials and methods}

\subsection{Plant materials and treatments}

Sweet potato (Pomoea batatas Lam. cv. Tainong 57) was grown at $28{ }^{\circ} \mathrm{C}$ in a growth chamber under $16 \mathrm{~L}$ $(06: 00-22: 00 \mathrm{~h}) / 8 \mathrm{D}(22: 00-06: 00 \mathrm{~h})$. For treatments with different photoperiods, the $16 \mathrm{~L} / 8 \mathrm{D}$ entrained plants were moved to separate growth chambers with 24-h light (LL), 24-h dark (DD) or other photoperiods. Leaf samples were harvested at time intervals indicated. Before treatments with various sugars and chemicals, leaf-petioles $(10 \mathrm{~cm})$ were cut from plants (about $20 \mathrm{~cm}$ in height) and cultured in $\mathrm{H}_{2} \mathrm{O}$ for 1 day under a dark condition (DD) to deplete endogenous sugars. These cuttings were then transferred and incubated in 175 $\mathrm{mM}$ of sugar solutions (or $6 \%$ of sucrose solution) [16] while maintaining in a dark growth chamber at $28{ }^{\circ} \mathrm{C}$. Since the maximum level of GBSSI gene expressions resulted from sucrose treatment was observed at $8 \mathrm{~h}$ (unpublished data), leaf samples were harvested at $8 \mathrm{~h}$ after sugar treatments. In order to evaluate effects of various inhibitors of photosynthesis, protein kinases and phosphatases on the sucrose-stimulated GBSSI expressions, leaf-petiole cuttings were pretreated with various inhibitors for $1 \mathrm{~h}$ before the sucrose treatment. Again, leaf samples were harvested at $8 \mathrm{~h}$ after sucrose treatment. Inhibitors were not removed during the 8-h period of sucrose treatment. All experiments were repeated at least twice using $\mathrm{H}_{2} \mathrm{O}$-cultured leaf-petiole cuttings as controls.

\subsection{RNA extraction from sweet potato leaves}

RNA was isolated from sweet potato leaves according to the procedure described by Yeh et al. [23]. Leaf sample $(0.2 \mathrm{~g})$ was ground in liquid nitrogen and the powder was mixed with $2 \mathrm{ml}$ of extraction buffer (7.5 M guanidine hydrochloride, $25 \mathrm{mM}$ sodium citrate, $\mathrm{pH} 5.2,0.5 \%[\mathrm{w} / \mathrm{v}]$ lauroyl sarcosine and $0.1 \mathrm{M} \beta$-mercaptoethanol). The mixture was then incubated at room temperature for $10 \mathrm{~min}$ before centrifugation at $12,000 \times g$ for $10 \mathrm{~min}$. The supernatant was treated in succession with equal volume of phenol:chloroform:isoamyl alcohol (25:24:1, v/v/v) and chloroform:isoamyl alcohol (24:1, v/v). RNA was precipitated with $5 \mathrm{ml}$ of $100 \%$ ethanol and $0.2 \mathrm{ml}$ of 3 $\mathrm{M}$ sodium acetate, $\mathrm{pH} 5.2$, at $-70{ }^{\circ} \mathrm{C}$ for $30 \mathrm{~min}$. After centrifugation, the pellet was dissolved in $0.5 \mathrm{ml}$ $\mathrm{H}_{2} \mathrm{O}$, and treated again with phenol:chloroform: isoamyl alcohol $(25: 24: 1, \mathrm{v} / \mathrm{v} / \mathrm{v})$ and chloroform: isoamyl alcohol $(24: 1, v / v)$ before precipitation. Finally, the RNA pellet was dissolved in $100 \mu 1 \mathrm{H}_{2} \mathrm{O}$. 


\subsection{Analysis by northern blotting}

Total RNAs $(10 \mu \mathrm{g})$ were separated on $1 \%$ formaldehyde-agarose gels [24]. The GBSSI cDNA probe [4] was radioactively labeled with $\alpha-{ }^{32} \mathrm{P}-\mathrm{dCTP}$ using a random primer labeling kit (Amersham, UK). After hybridization, the membranes were washed twice with $2 \times \mathrm{SSC}$ (1 1 of $20 \times \mathrm{SSC}$ stock solution contained $175.3 \mathrm{~g}$ of $\mathrm{NaCl}$ and $88.2 \mathrm{~g}$ of sodium citrate, $\mathrm{pH} 7.0$ ) containing $0.1 \%$ (w/v) SDS at room temperature for $30 \mathrm{~min}$ and twice with $0.1 \times \mathrm{SSC}$ containing $0.1 \%(\mathrm{w} / \mathrm{v}) \mathrm{SDS}$ at $55^{\circ} \mathrm{C}$ for $30 \mathrm{~min}$ [24]. EtBr-stained 28S rRNA patterns were used as internal standards in all northern blot analysis data. Accumulation of GBSSI mRNA were quantified from the northern blot using Nobel ABC-Tiger Gel Documentation and Analysis System version 2.0 (Taigen, Taiwan). Relative levels were based on the measurement determined for the sample of leaf-petiole cuttings that were cultured in $\mathrm{H}_{2} \mathrm{O}(100 \%)$.

\subsection{Isolation and immunoblotting of starch granule-bound proteins}

Leaves (5 g) were powdered in liquid nitrogen and subsequently ground with $25 \mathrm{ml}$ of extraction buffer (50 mM Tris-HCl, pH 7.4, $2 \mathrm{mM}$ EDTA and $1 \mathrm{mM}$ DTT). The sample mixture was then filtered through two layers of Micracloth (Calbiochem, La Jolla, CA) before centrifuging at $5000 \times g$ for $5 \mathrm{~min}$. The pellet was resuspended with $0.5 \mathrm{ml}$ of gradient buffer $(50 \mathrm{mM}$ Tris- $\mathrm{HCl}, \mathrm{pH}$ 7.4, $2 \mathrm{mM}$ EDTA, $1 \mathrm{mM}$ DTT and 350 $\mathrm{mM}$ sorbitol), and loaded onto a discontinuous Percoll density gradient (80, 60 and 40\%; 80\% Percoll solution contained $80 \%$ [v/v] Percoll, $0.6 \mathrm{mM}$ glutathione [reduced form, GSH], 2.4\% [w/v] polyethylene glycol $\left[M_{\mathrm{W}}\right.$ 4000], 0.8\% [w/v] BSA, 0.8\% [w/v] Ficoll, 20\% [v/v] $5 \times$ gradient buffer). After centrifuging at $7000 \times g$ for $15 \mathrm{~min}$, the starch granule pellet was washed by cold acetone and then air-dried. Starch granule-bound proteins were extracted from starch granules by SDSsample buffer [25]. Proteins were separated on a $7.5 \%$ SDS-polyacrylamide gel and transferred onto a nitrocellulose membrane (Amersham, Buckinghamshire, UK) for the western blot analysis using an antibody raised against the potato GBSSI (kindly provided by Dr R. Visser).

\subsection{Starch measurements}

A leaf sample $(0.5 \mathrm{~g})$ was ground in liquid nitrogen, then the powdered sample was washed by shaking with $70 \%$ ethanol at room temperature. After washing, the sample was centrifuged at $13,000 \times g$, and the pellet was analyzed for starch by the test-combination for carbohydrate (Boehringer Mannheim, Germany) according to the instruction. All measurements were repeated three times using different leaf samples, and their mean values were presented.

\section{Results}

\subsection{Effect of light on circadian rhythm of GBSSI expressions}

In order to evaluate whether or not the biological clock was set by light, some plants maintained under 16 $\mathrm{L} / 8 \mathrm{D}$ with light turning on at 06:00 $\mathrm{h}$ were moved to a separate growth chamber under the same photoperiod but with the light turning on at 18:00 $\mathrm{h}$ for a period of 14-days adaptation. Northern blot analysis showed that the resetting of time for light-dark transition did cause a shift in the accumulation peak of GBSSI mRNA. For the control, when light was turned on at $06: 00 \mathrm{~h}$, the peak of GBSSI mRNA accumulation was observed at 10:00 $\mathrm{h}$, then the level of mRNA decreased gradually until almost completely disappeared at 02:00 h. However, the transcript reappeared at end of the photoperiod (Fig. 1A). When the time of light-dark transition was reset with light turning on at 18:00 h, the rhythmic pattern of GBSSI mRNA accumulations was similar to that of the control, but the peak of accumulation appeared at 22:00 $\mathrm{h}$ (Fig. 1B). However, in either case, the peak of
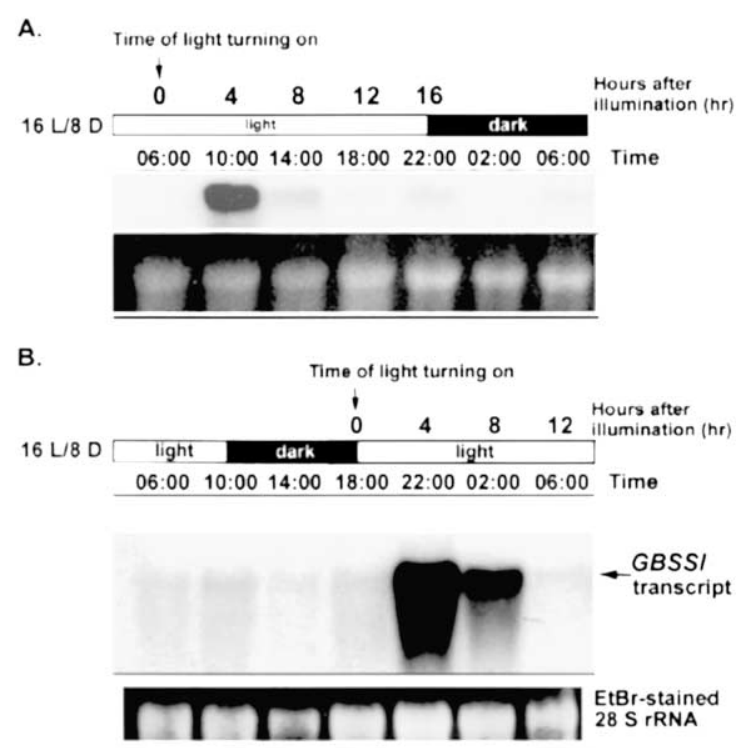

Fig. 1. Effects of resetting the time of light-dark transition on GBSSI gene expressions during a diurnal cycle. (A) Plants were maintained in a growth chamber under $16 \mathrm{~L} / 8 \mathrm{D}$ with light turning on at 06:00 h. Leaves were harvested at 4-h intervals. (B) Some plants originally maintained in the condition A were moved to a separate chamber under $16 \mathrm{~L} / 8 \mathrm{D}$, but the time of light turning on was shifted from the regular 06:00-18:00 $\mathrm{h}$ and these plants were entrained for 14 days before leaves were harvested at 4 -h intervals. Total RNAs $(15 \mu \mathrm{g}$ per lane) were probed with the $\alpha-{ }^{32} \mathrm{P}$ labeled GBSSI cDNA. 


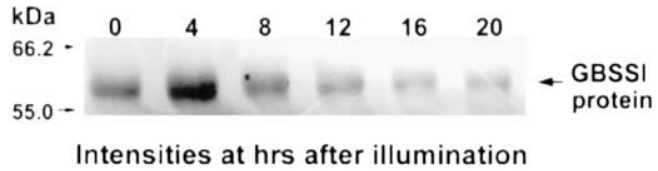

Fig. 2. Accumulation patterns of GBSSI protein during a diurnal cycle in leaves under $16 \mathrm{~L} / 8 \mathrm{D}$. Granule-bound proteins were isolated from starch granules $(5 \mathrm{mg})$ of leaves that were harvested at 4-h intervals. Proteins were separated on a $7.5 \%$ SDS-PAGE gel and immunoblotted with antiserum against the GBSSI of potato.

GBSSI mRNA accumulations always appeared in $4 \mathrm{~h}$ after the light was turned on.

\subsection{Changes in accumulation patterns of GBSSI protein and starch in leaves during diurnal cycles}

Under the $16 \mathrm{~L} / 8 \mathrm{D}$ photoperiod (light was turned on at 06:00 h), changes in GBSSI protein levels were observed clearly in the leaf tissue (Fig. 2). The peak of GBSSI protein accumulation was observed in $4 \mathrm{~h}$ after the light was turned on, then the level of protein decreased gradually. Similarly, the amount of starch accumulated rapidly after illumination and decreased during the dark period (Fig. 3A). When the light period was prolonged to $24 \mathrm{~h}$ in a separated experiment, the pattern of starch accumulation was also similar to that of the control (Fig. 3B). The amount of starch still declined at the usual dark period, even though the illumination was continued. Under the DD environment, starch content also increased in $4 \mathrm{~h}$ and decreased quickly until the end of the cycle, and the level of starch in this condition was lower than that of the 16 L/8 D or LL (Fig. 3C).

\subsection{Effects of sugars on GBSSI gene expressions}

When leaf-petiole cuttings were treated with $175 \mathrm{mM}$ sorbitol or sucrose, only sucrose produced a significant stimulation in the accumulation of GBSSI transcripts based on the northern blot analysis (Fig. 4A, D). Since 8 h-treatments with sorbitol could not cause an increase in GBSSI mRNA accumulation, the sucrose-stimulated expression of GBSSI transcripts was not a result of osmotic effect. In order to study whether hydrolysis of sucrose is required for the stimulation of GBSSI expression, leaf-petiole cuttings were cultured in $175 \mathrm{mM}$ glucose, fructose, or their combination. The result showed that glucose or fructose alone was ineffective in stimulating the accumulation of GBSSI mRNA (Fig. 4B, D). However, the accumulation was enhanced by a combination of glucose and fructose at a concentration of $87.5 \mathrm{mM}$ for each hexose (Fig. 4B, D). A treatment of cuttings with glucosamine, an inhibitor for hexokinase, did not reduce the accumulation of GBSSI transcripts (Fig. 4C, D). On the contrary, glucosamine produced an effect stronger then that of the sucrose treatment, and both sucrose and glucosamine might have an additive effect in stimulating the expression of GBSSI gene (Fig. 4C, D).

\subsection{Effects of inhibitors for protein kinases and phosphatases on the sucrose-stimulated GBSSI expressions}

Fig. 5A and $\mathrm{C}$ shows that a pretreatment for $1 \mathrm{~h}$ with $50 \mu \mathrm{M}$ or $100 \mu \mathrm{M}$ cycloheximide (CHX) had no effect on the expression of GBSSI gene stimulated by sucrose. Previous experiments indicated that leaf-petiole cuttings pretreated with $50 \mu \mathrm{M}$ CHX for $1 \mathrm{~h}$ was effective to inhibit de novo synthesis of proteins (unpublished

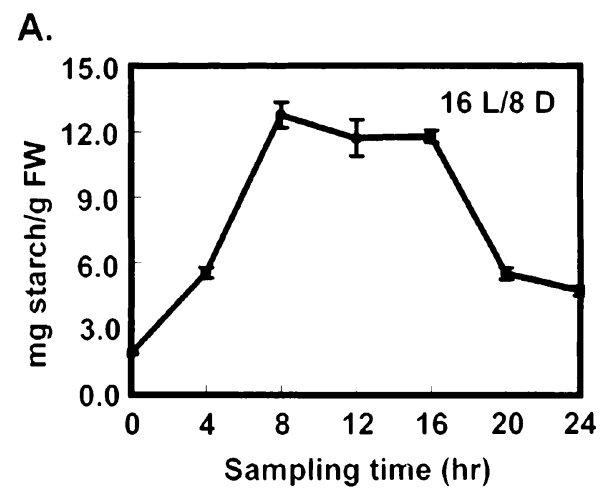

B.

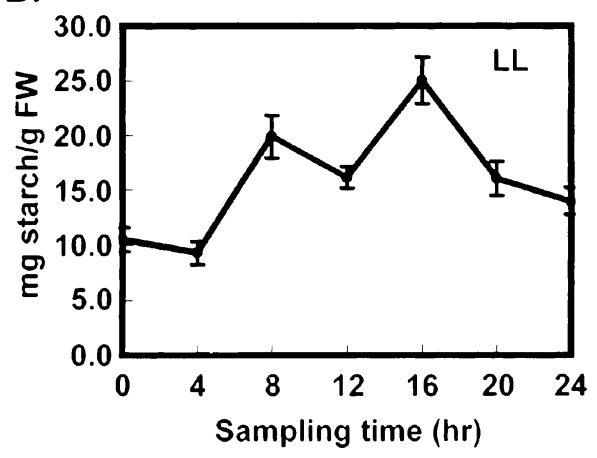

C.

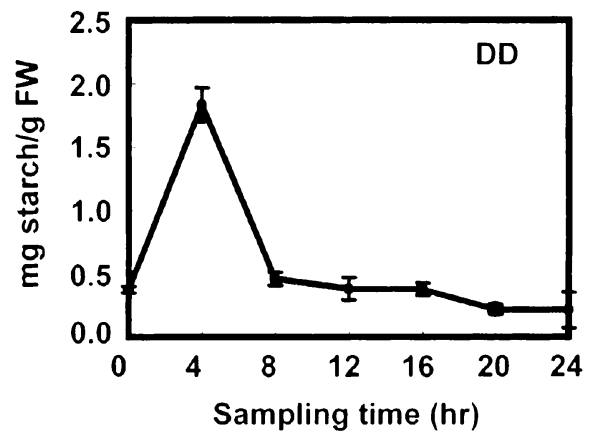

Fig. 3. Patterns of starch accumulation in leaves treated with different photoperiods. Starch contents were determined under 16 L/8 D (A), LL (B) and DD (C) conditions. 


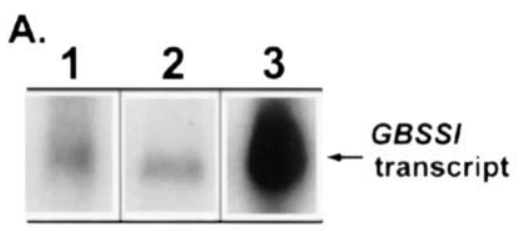

B.

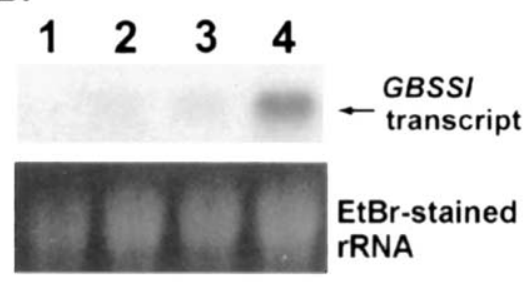

C.

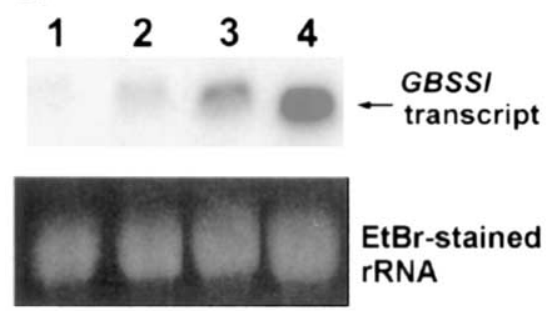

D.

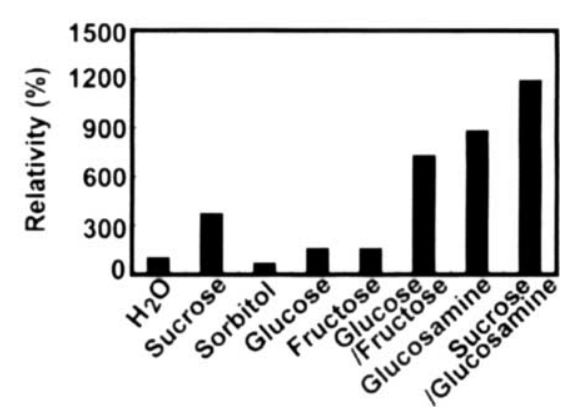

Fig. 4. Effects of various sugars and hexokinase inhibitor on GBSSI expressions. Leaf-petioles were cultured with $\mathrm{H}_{2} \mathrm{O}$ in DD for 1 day before treating with $175 \mathrm{mM}$ of sugar solutions. After treatments for $8 \mathrm{~h}$, leaves were harvested and RNA extracted. GBSSI mRNA was determined by the northern blot hybridization. (A) Lane $1, \mathrm{H}_{2} \mathrm{O}$; lane 2, $175 \mathrm{mM}$ sorbitol; lane 3, $175 \mathrm{mM}$ sucrose. (B) Lane 1, $\mathrm{H}_{2} \mathrm{O}$; lane 2, $175 \mathrm{mM}$ glucose; lane 3, $175 \mathrm{mM}$ fructose; lane 4, a combination of $87.5 \mathrm{mM}$ glucose and $87.5 \mathrm{mM}$ fructose. (C) Effect of hexokinase inhibitor on the sucrose-stimulated GBSSI expression. Lane 1, leafpetiole cuttings were kept in $\mathrm{H}_{2} \mathrm{O}$ as a control. Lane 2, cuttings were cultured in $175 \mathrm{mM}$ sucrose solution. Lane 3, cuttings were treated with $175 \mathrm{mM}$ glucosamine. Lane 4, leaf-petiole cuttings were pretreated with $175 \mathrm{mM}$ of glucosamine for $1 \mathrm{~h}$ before $175 \mathrm{mM}$ of sucrose was added into the solution and cultured for $8 \mathrm{~h}$. D. Data in (A), (B) and (C) were quantified by a densitometer. Relative levels were based on the measurement determined for the $\mathrm{H}_{2} \mathrm{O}$-cultured leaf-petiole cuttings $(100 \%)$.

data). Therefore, the lack of effect on the sucrose-stimulated GBSSI expression was not the result of inability to take up CHX in the cutting. In order to analyze whether protein dephosphorylation was involved in the signal transduction pathway of sucrose-stimulated $G B$ $S S I$ expressions, effects of protein phosphatase 1 and
$2 \mathrm{~A}$ inhibitors were examined. Results showed that the sucrose-stimulated expression of GBSSI was not inhibited by cantharidin, but was completely inhibited by okadaic acid (Fig. 5B, C). This inhibitory effect of okadaic acid was not attributable to DMSO, the solvent used for cantharidin and okadaic acid, since
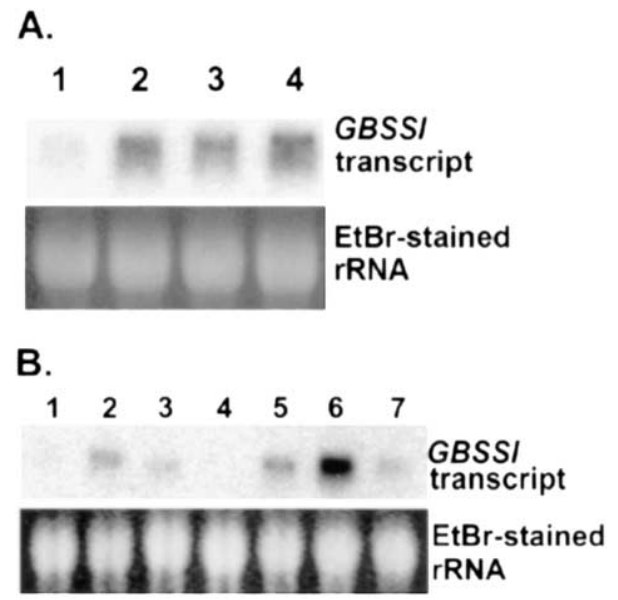

C.

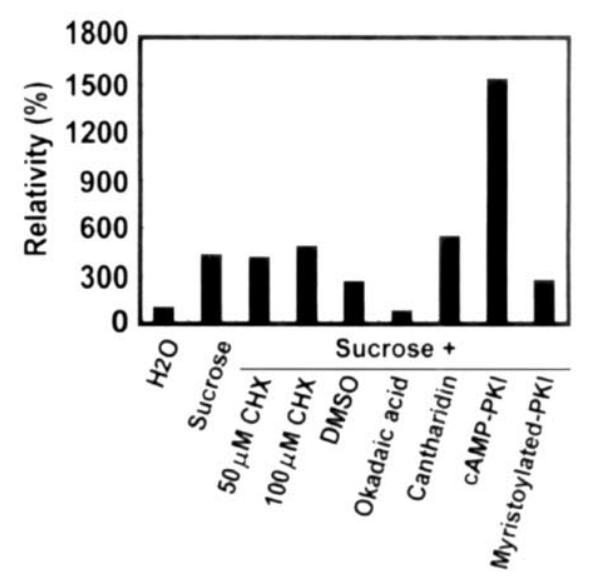

Fig. 5. Northern blot analysis of the sucrose-stimulated GBSSI expressions following treatments with cycloheximide (CHX), protein kinase or phosphatase inhibitors. (A) Effect of CHX on the sucrosestimulated GBSSI gene expressions. Lane 1, leaf-petiole cuttings were maintained in $\mathrm{H}_{2} \mathrm{O}$ as a control. Lane 2, cuttings were treated with $175 \mathrm{mM}$ sucrose for $8 \mathrm{~h}$. Lane 3, leaf-petiole cuttings were pretreated for $1 \mathrm{~h}$ with $50 \mu \mathrm{M}$ of $\mathrm{CHX}$ before $175 \mathrm{mM}$ of sucrose was added. Lane 4 , leaf-petiole cuttings were pretreated for $1 \mathrm{~h}$ with $100 \mu \mathrm{M}$ of CHX before $175 \mathrm{mM}$ of sucrose was added. (B) Leaf-petiole cuttings were pretreated for $1 \mathrm{~h}$ with protein phosphatase inhibitors or protein kinase inhibitors (PKI) before $175 \mathrm{mM}$ of sucrose was added into cultured solutions. Lane $1, \mathrm{H}_{2} \mathrm{O}$; lane 2, $175 \mathrm{mM}$ sucrose only; lane 3, $0.1 \%(\mathrm{v} / \mathrm{v}) \mathrm{DMSO} / 175 \mathrm{mM}$ sucrose; lane $4,1 \mu \mathrm{M}$ okadaic acid/175 $\mathrm{mM}$ sucrose; lane $5,1 \mu \mathrm{M}$ cantharidin $/ 175 \mathrm{mM}$ sucrose; lane $6,1 \mu \mathrm{M}$ cAMP-dependent PKI/175 mM sucrose; lane 7, $1 \mu \mathrm{M}$ myristoylateddependent PKI/175 mM sucrose. RNAs were extracted from leaves after $8 \mathrm{~h}$ of treatments with sucrose and different chemicals. GBSSI mRNA was determined by the northern blot hybridization. (C) Data in (A) and (B) were quantified by a densitometer. Relative levels were based on the measurement determined for the $\mathrm{H}_{2} \mathrm{O}$-cultured leaf-petiole cuttings $(100 \%)$. 
DMSO alone had only a slight effect on the sucrosestimulated expression of GBSSI (Fig. 5B, C). In addition, protein phosphorylation might also be involved in this signal transduction pathway. Although myristoylated protein kinase $\mathrm{C}$ inhibitor produced a slight inhibitory effect on the sucrose-stimulated GBSSI expression, cAMP-dependent protein kinase inhibitor enhanced the expression (Fig. 5B, C).

\subsection{Effect of sucrose on the circadian expression of GBSSI gene}

In order to determine whether or not sucrose was involved in the signal transduction pathway for the circadian expression of GBSSI, the following experiments with sucrose treatments were carried out under $16 \mathrm{~L} / 8 \mathrm{D}$. First, all leaf-petiole cuttings were cultured in $\mathrm{H}_{2} \mathrm{O}$ under $16 \mathrm{~L} / 8 \mathrm{D}$ for 7 days, then leaf-petiole cuttings were moved to $175 \mathrm{mM}(6 \%)$ sucrose solution at the beginning of a dark cycle on the 7th day until all samples were harvested at indicated time points on the 8th day (see also Fig. 6B). Accumulations of GBSSI mRNA were enhanced at all sampling times and the circadian rhythm was also maintained (Fig. 6B). In a second experiment to further evaluate the sucrose effect, leaf-petiole cuttings were also transferred to sucrose solution, but only for $8 \mathrm{~h}$. Subsequently, these cuttings were moved back to $\mathrm{H}_{2} \mathrm{O}$ at the beginning of 8 th day before leaves were harvested at indicated time points. Results indicated that the removal of sucrose produced no effect on the circadian rhythm of GBSSI mRNA accumulations; however, the sucrose-enhanced effect on transcription was reduced gradually (Fig. 6C).

\subsection{Effects of photosynthesis on GBSSI gene expressions}

In order to minimize the effect of endogenous sucrose, $\mathrm{H}_{2} \mathrm{O}$-cultured leaf-petiole cuttings were moved to DD on the 8th day to deplete endogenous sucrose after $16 \mathrm{~L} / 8 \mathrm{D}$ entraining for 7 days. Then, leaf-petiole cuttings were transferred to a sucrose solution at the beginning of 9th day while maintaining under DD condition. Leaf samples were harvested after sucrose treatment for $4 \mathrm{~h}(10: 00 \mathrm{~h})$ and $12 \mathrm{~h}(18: 00 \mathrm{~h})$, respectively (Fig. 7A). Northern blot analysis revealed a similar pattern to that was observed under $16 \mathrm{~L} / 8 \mathrm{D}$, indicating that GBSSI mRNA accumulations were enhanced by sucrose, but sucrose had no effect on the rhythmic pattern. When leaf-petiole cuttings were kept under DD condition, the expression of GBSSI was very weak (Fig. 7B, lane 1); however, this dark effect could be reversed by illumination and resulted in a large accumulations of GBSSI transcripts (Fig. 7B, lane 2). When leaf-petiole cuttings were pretreated under DD for $1 \mathrm{~h}$ with the 3-(3,4-dichlorophenyl)-1,1-dimethy-

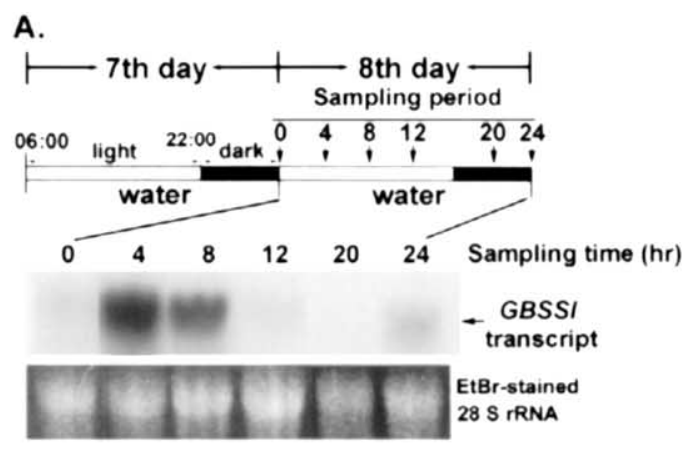

B.

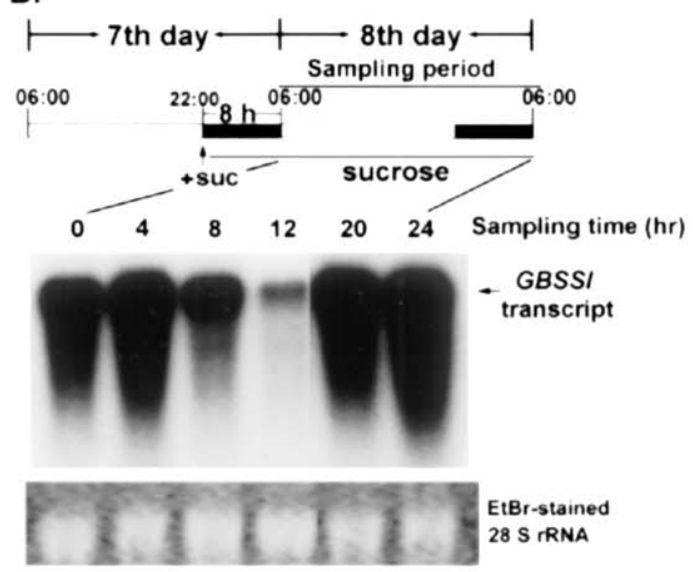

C.

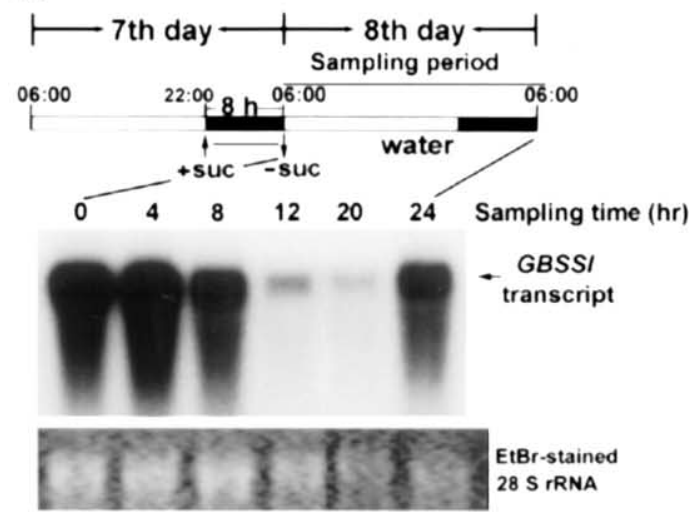

Fig. 6. Effects of sucrose on GBSSI gene circadian expressions under $16 \mathrm{~L} / 8 \mathrm{D}$ condition. (A) $16 \mathrm{~L} / 8 \mathrm{D}$-entrained leaf-petiole cuttings were incubated in $\mathrm{H}_{2} \mathrm{O}$ for 8 days as a control, and leaves were harvested at the indicated time points on the 8 th day. (B) $16 \mathrm{~L} / 8 \mathrm{D}$-entrained leaf-petiole cuttings were incubated in $\mathrm{H}_{2} \mathrm{O}$ for 7 days and then transferred to $175 \mathrm{mM}(6 \%)$ sucrose solution at the beginning of dark period on the 7th day before leaf samples were harvested on the 8th day. During the entire harvesting period, leaf-petiole cuttings were kept in the sucrose solution. (C). Leaf-petiole cuttings were treated in a fashion identical to the condition described under (B), except that these cuttings were transferred back to $\mathrm{H}_{2} \mathrm{O}$ on the 8th day at 06:00 h, i.e. after $8 \mathrm{~h}$ of sucrose-treatment during the night of 7 th day. Total RNAs ( $5 \mu \mathrm{g}$ for each sample) were separated on a $1 \%$ formaldehyde-agarose gel, and GBSSI mRNA was detected using the $\alpha_{-}{ }^{32} \mathrm{P}$ labeled GBSSI cDNA probe. 
lurea (DCMU), an inhibitor for the transport of photosynthetic electrons, before these cuttings were illuminated for $8 \mathrm{~h}$, accumulations of GBSSI mRNA were reduced (Fig. 7B, lane 3). When the DCMU treatment was kept continuously for 3 days under a continuous light condition, the expression of GBSSI was similar to that observed in leaves treated under the DD condition (Data not shown).

When leaf-petiole cuttings were entrained in $16 \mathrm{~L} / 8$ $\mathrm{D}$ condition (light was turned on at $06: 00 \mathrm{~h}$ and turned off at 22:00 h) for 7 days, the expression of GBSSI showed a pattern typical of circadian fluctuation. The peak of GBSSI mRNA accumulation was observed at 4 $\mathrm{h}(10: 00 \mathrm{~h})$ after the light was turned on, and the level of GBSSI transcripts was greatly decreased at $12 \mathrm{~h}$ $(18: 00 \mathrm{~h})$ (Fig. 7C, lanes $1-3)$. In a similar experiment to demonstrate that DCMU had no effect on the circadian rhythm, some leaf-petiole cuttings were moved to DD for 1 day to deplete endogenous sugars, and then these cutting were pretreated with DCMU for $1 \mathrm{~h}$ before transferring back to the $16 \mathrm{~L} / 8 \mathrm{D}$ condition.

A.

\begin{tabular}{|c|c|c|}
\hline \multicolumn{2}{|c|}{ dark } & \\
\hline water & $6 \%$ sucrose & \\
\hline $10: 00 \quad 18: 00$ & $10: 00 \quad 18: 00$ & Sampling time \\
\hline$=-$ & and & $\begin{array}{l}\text { GBSSI } \\
\text { transcript }\end{array}$ \\
\hline & & $\begin{array}{c}\text { EtBr-stained } \\
\text { 28S rRNA }\end{array}$ \\
\hline
\end{tabular}

B.
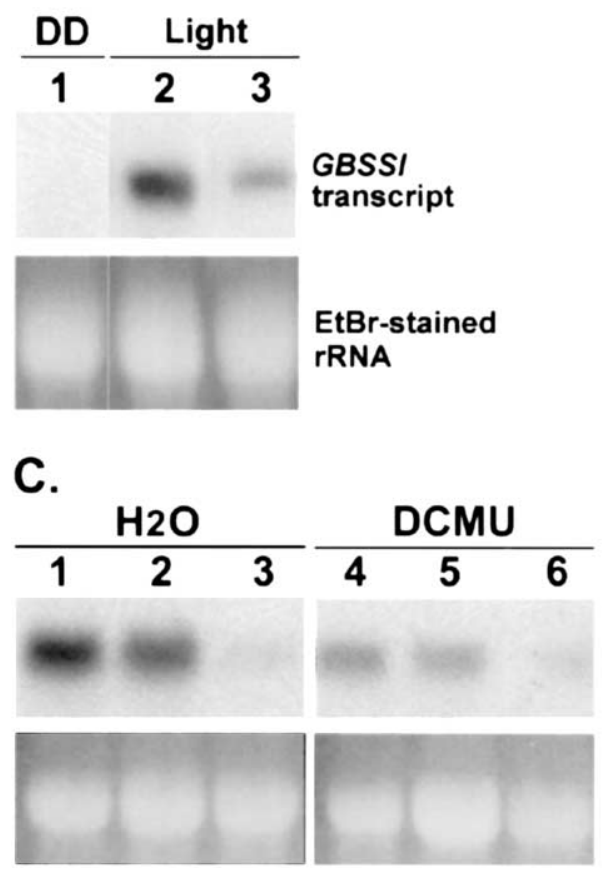

Fig. 7. (Continued)
As expected, the northern blot analysis showed that DCMU reduced the amount of GBSSI transcript but had no effect on the pattern of circadian expression (Fig. 7C, lanes 4-6).

\section{Discussion}

Our previous study showed that levels of GBSSI mRNA accumulation in sweet potato leaves exhibited a circadian-regulated pattern [4]. This experiment was repeated here for the purpose of comparisons with effects of time resetting for light-dark transition. One of the features of circadian rhythms is entrainability, and factors involved in entraining are light and temperature [26]. In an effort to demonstrate that light is responsible for setting the rhythm of GBSSI expressions, the time of light-dark transition was reset and plants were entrained under the new condition for 14 days. The result showed that the peak of GBSSI gene expression was indeed shifted. However, the accumulation peak of GBSSI transcript still occurred in $4 \mathrm{~h}$ after illumination regardless of the time when the light was turned on (Fig. 1). These results clearly demonstrate that the clock, which controlled the expression of GBSSI gene, was set by light. This hypothesis was also supported by a study on suspension cells of sweet potato [27]. In a dark-adapted suspension cell culture, expressions of GBSSI gene did not show circadian rhythm, but the situation could be reversed by light-treatment and pho-

Fig. 7. Effects of sucrose and photosynthesis on GBSSI gene expressions. (A) Effects of sucrose on GBSSI gene expressions under a DD condition. Leaf-petiole cuttings were moved to DD on the 8 th day after 7 days of entraining under $16 \mathrm{~L} / 8 \mathrm{D}$ in $\mathrm{H}_{2} \mathrm{O}$ with light turning on at $06: 00 \mathrm{~h}$. At 06:00 $\mathrm{h}$ on the 9th days ( 1 day after DD treatment), some cuttings were transferred to $175 \mathrm{mM}(6 \%)$ sucrose solution while other cuttings were maintained in $\mathrm{H}_{2} \mathrm{O}$ as a control. RNAs were prepared from leaves harvested at 10:00 h $(4 \mathrm{~h}$ after sucrose treatment) and 18:00 h (12 h after sucrose treatment), respectively. (B) Effect of DCMU on the accumulation level of GBSSI mRNA in light. All leaf-petiole cuttings were cultured with $\mathrm{H}_{2} \mathrm{O}$ in DD for 1 day before various treatments. Lane 1, cuttings were continuously kept in DD for $8 \mathrm{~h}$ before leaves were harvested. Lane 2, cuttings were illuminated for $8 \mathrm{~h}$ before leaves were harvested. Lane 3, cuttings were pretreated with $500 \mu \mathrm{M}$ of DCMU for $1 \mathrm{~h}$ before illumination for $8 \mathrm{~h}$, and DCMU was not removed during the period of illumination. (C) Effect of DCMU on the circadian expression of GBSSI gene under $16 \mathrm{~L} / 8 \mathrm{D}$. Leaf-petiole cuttings were entrained under $16 \mathrm{~L} / 8 \mathrm{D}$ (light was turned on at $06: 00 \mathrm{~h}$ and turned off at 22:00 h) for 7 days. Samples in lanes 1-3 were maintained under 16 $\mathrm{L} / 8 \mathrm{D}$, and leaves were harvested at times indicated right after the entrained period. Sampling time were 10:00 h (lane 1), 14:00 h (lane 2) and 18:00 h (lane 3), respectively. Some of leaf-petiole cuttings were moved to DD for 1 day to deplete endogenous sucrose after the entrained period, and then the cuttings were pretreated with DCMU for $1 \mathrm{~h}$ before transferring back to the $16 \mathrm{~L} / 8 \mathrm{D}$ condition. Leaves were then harvested at the indicated times (lane 4, 10:00 h; lane 5, 14:00 h; lane 6, 18:00 h). Expressions of GBSSI gene were determined by the northern blot hybridization. 
toperiod entraining [27]. Like GBSSI mRNA, the accumulation of GBSSI protein also exhibited a circadian pattern (Fig. 2). Since the GBSSI was not detected in soluble fractions, the fluctuation of GBSSI protein in starch granules was not the result of GBSSI re-distribution between granule-bound and soluble fractions. As expected, starch accumulation pattern was also circadian-fluctuated (Fig. 3). The circadian regulations of starch synthetic rate or starch accumulation were also observed in sugar beet and snapdragon [5,13], and the fluctuation in starch accumulation was suggested as a result of changes in synthetic activities [13].

In addition to regulation by a circadian clock, the expression of GBSSI gene in sweet potato was also regulated by sucrose. Levels of sucrose in sweet potato leaves were significantly lower under DD than $16 \mathrm{~L} / 8 \mathrm{D}$ conditions, and the accumulation of GBSSI mRNA was also reduced under this condition (data not shown). Furthermore, the level of GBSSI mRNA could be enhanced by supplementing with sucrose (Fig. 4). These observations clearly suggest that sucrose function as a stimulator for the transcription of GBSSI gene. This result is in agreement with a previous study using promoter assay, which indicated that expression of GBSSI gene could be enhanced by sugars in potato [17]. The stimulation of GBSSI gene expressions in leaves of sweet potato by sucrose was not the result of an osmotic effect because treatments with sorbitol and hexose were ineffective (Fig. 4A, B and D).

Sucrose has been shown to function as an indirect signal to regulate gene expressions since it could be replaced by lower concentrations of glucose or fructose [19]. However, present studies indicate that, in 8-h of treatments, glucose or fructose alone could not enhance the accumulation of GBSSI transcripts (Fig. 4B, D). This observation suggests that sucrose may function as a specific regulator for GBSSI expressions in sweet potato. This hypothesis was supported by the observation that a treatment with combination of glucose and fructose stimulated the expression of GBSSI gene to a degree much higher than with glucose or fructose alone (Fig. 4B, D), presumably, the combination facilitated the synthesis of sucrose inside the leaf tissue. When glucose treatment was prolonged to $24 \mathrm{~h}$, accumulation of GBSSI transcripts in sweet potato leaves was also enhanced but still less than that of the sucrose-treated leaves (data not shown). It is not clear whether this 'glucose effect' due to a prolonged treatment is the result of isomerization of the hexose sugar coupling with a re-synthesis of sucrose or the prolonged treatment with glucose might cause an osmotic stress to induce an accumulation of sucrose. In Arabidopsis seedlings, synthesis of sucrose was activated by glucose added in the incubation medium [28]. Also, osmotic stress is known to induce sucrose accumulation in plant tissues. For example, sorbitol-induced osmotic stress and caused an accumulation of sucrose in sweet potato suspension cells [29]. A prolonged treatment of sweet potato leaf-petiole cuttings with sorbitol for $24 \mathrm{~h}$ did enhance accumulation of GBSSI mRNA (data not shown). It should also be noted that enhancements of GBSS and branching enzyme gene expressions in cassava were observed to be stimulated by culturing in glucose for $24 \mathrm{~h}$ in a dark condition [18]. In potato, expressions of GBSSI gene could also be enhanced when leaf explants were cultured in fructose or glucose [17].

The sugar sensing for GBSSI expression was not mediated by hexokinase pathway because accumulations of GBSSI transcripts could not be stimulated by glucose or fructose alone (Fig. 4B, D) and glucosamine did not block the sucrose effect on GBSSI gene expressions (Fig. 4C, D). This possibility was further supported by the observation that increases in GBSSI transcripts could be resulted from a treatment with glucose and fructose combination (Fig. 4B, D), presumably, the combination promoted the synthesis of sucrose to activate an intracellular sensor for stimulating the expression of GBSSI gene.

In addition to GBSSI, several other genes in sweet potato, e.g. sporamin, ADP-glucose pyrophosphorylase and $\beta$-amylase were also found to be responding to sucrose for expressions, and phosphorylation and dephosphorylation of several proteins were involved $[16,30]$. The observation that CHX was ineffective to affect the sucrose-regulated GBSSI gene expressions (Fig. 5A, C) suggests that de novo protein synthesis was not necessary for regulating the transcription of GBSSI gene. Therefore, modifications of pre-existed proteins might be involved in the signal transduction of sucroseregulated GBSSI transcription. Okadaic acid, a selective inhibitor of protein phosphatase types 1 and $2 \mathrm{~A}$ [31], inhibited the sucrose-stimulated GBSSI gene expression strongly (Fig. 5B, C). This inhibition suggests either an involvement of dephosphorylation in the transduction pathway or a block in sucrose uptake, since okadaic acid was also shown to block the function of sucrose transporters to inhibit sucrose uptake in sugar beet [32]. However, the observations that the level of GBSSI mRNA was enhanced by treating with glucose and fructose combinations, but the enhancement was inhibited by okadaic acid (data not shown), suggest that the decrease of sucrose-stimulated GBSSI expressions by okadaic acid might not be attributable to an inhibition in sucrose uptake. Instead, it is more likely that the decrease in expression was due to the result of a block in the internal signal cascade of sucrose-regulated pathway, presumably involving phosphatases. Cantharidin at low concentrations will inhibit protein phosphatase 2A, but not protein phosphatase 1 [33]. The observation that cantharidin at the concentration tested was ineffective to inhibit the sucrose-stimulated 
GBSSI transcription (Fig. 5B, C) suggests that protein phosphatase type 1 was involved in the signal transduction of sucrose-regulated GBSSI expressions.

In addition to dephosphorylation, phosphorylation might also be involved in the signal transduction pathway, because myristoylated protein kinase $\mathrm{C}$ peptide inhibitor, a specific inhibitor for calcium- and phospholipid-dependent protein kinase $\mathrm{C}$, was found to reduce slightly the level of sucrose-induced GBSSI transcripts (Fig. 5B, C). On the contrary, cAMP-dependent protein kinase inhibitor enhanced sucrose-regulated GBSSI transcriptions (Fig. 5B, C). The different effects of protein kinase inhibitors on the sucrose-stimulated $G B$ $S S I$ expressions suggest that calcium-, phospholipid-, or cAMP-dependent protein kinase $\mathrm{C}$ might be involved in the pathway of sucrose regulation on GBSSI expressions. Since expressions of genes related to carbohydrate metabolism could be affected by inhibitors of protein kinase and phosphatase, which might result in changes in levels of metabolic intermediates; in turn, these changes might produce an indirect effect on $G B$ $S S I$ gene expressions, especially since GBSSI is the final enzyme in the biosynthetic pathway of starch. Therefore, we could not rule out the possibility that changes in GBSSI expression due to inhibitor treatments were measurements of indirect effects.

Although sucrose may function as a stimulator for the transcription, the circadian fluctuation of GBSSI mRNA under any photoperiods might not be affected by sucrose. This hypothesis was supported by the observation that the circadian pattern of accumulating GBSSI transcripts could be maintained for at least three cycles under DD conditions, although the amplitude of rhythms was significantly lower than that of the $16 \mathrm{~L} / 8 \mathrm{D}$ (data not shown). When the exogenous sucrose was added and subsequently removed to change the sucrose concentration of the cultured condition, the rhythm of GBSSI gene expression was also maintained (Fig. 6C). As expected, expressions of GBSSI gene was enhanced by exogenous sucrose under DD conditions, but the circadian expressions of this gene was not changed (Fig. 7A). In addition, a treatment with photosynthetic inhibitor DCMU to prevent the synthesis of sucrose reduced the level of GBSSI transcripts (Fig. 7B), but it did not change the circadian rhythm (Fig. 7C). These results suggest that the pathways of circadian regulation and sucrose effects on GBSSI transcription are independent.

It is not clear from this study whether changes in starch accumulation were directly related to the circadian pattern of GBSSI and its regulation by sucrose since this enzyme is responsible primarily for amylose synthesis; however, other starch synthetic genes such as ADP-glucose pyrophosphorylase in Arabidopsis and potato leaves were also shown to be regulated by sucrose and light [15,34]. These observations might suggest that supply and availability of photosynthetic assimilates could serve as an important factor for controlling transitory starch metabolism in the source tissue during a diurnal cycle. Conceivably during the day, leaves should accumulate higher levels of sucrose than in the dark due to an active photosynthesis. Accordingly, greater amounts of mRNAs and proteins for GBSSI and other related genes for starch synthesis could be produced to promote conversion of sucrose to starch. Concomitantly, the situation should prevent a feedback inhibition on photosynthesis and osmotic stress. Thus, this sucrose regulation coupling with circadian rhythm should greatly facilitate carbons distribution from the source to sink tissues and prevent the leaf tissue from continuously accumulating starch.

In conclusion, we have demonstrated that light appears to regulate $G B S S I$ gene expression through two independent pathways: (1) light plays an input signal to set an endogenous oscillator which controls circadian expressions of GBSSI gene; (2) light is an indirect stimulator that quantitatively affects GBSSI mRNA levels mediating by the photosynthetic product, sucrose. The sugar sensing of the sucrose-regulated GBSSI expression was different from the hexokinase-mediated pathway. In addition, phosphorylated/dephosphorylated modifications of proteins might be involved in the transduction pathway for regulating the expression of GBSSI.

\section{Acknowledgements}

We are grateful to Dr Richard G.F. Visser for his kind gifts of potato GBSSI antibodies, and to Dr Yuh-Jang Shieh at the Institute of Botany, Academia Sinica in Taipei for his assistance on analysis of photosynthetic rate that were affected by DCMU-treatments. This research was supported by a grant NSC 89-2311B-002-007 from the National Science Council of the Republic of China.

\section{References}

[1] O.E. Nelson, C.Y. Tsai, Glucose transfer from adenosine diphosphate-glucose to starch in preparations of Waxy seeds, Science 145 (1964) 1194-1195.

[2] C.Y. Tsai, The function of the Waxy locus in starch synthesis in maize endosperm, Biochem. Genet. 11 (1974) 83-96.

[3] J.H.M. Hovenkamp-Hermelink, E. Jacobsen, A.S. Ponstein, R.G.F. Visser, G.H. Vos-Scheperkeuter, E.W. Bijmolt, J.N. de Vries, B. Witholt, W.J. Feenstra, Isolation of an amylose-free starch mutant of the potato (Solanum tuberosum L.), Theor. Appl. Genet. 75 (1987) 217-221.

[4] S.J. Wang, K.W. Yeh, C.Y. Tsai, Molecular characterization and expression of a starch granule-bound starch synthase gene in the sink and source tissues of sweet potato, Physiol. Plant. 106 (1999) 253-261. 
[5] A. Mérida, J.M. Rodríguez-Galán, C. Vincent, J.M. Romero, Expression of the granule-bound starch synthase I (Waxy) gene from snapdragon is developmentally and circadian clock regulated, Plant Physiol. 120 (1999) 401-409.

[6] W.S. Hillman, Biological rhythms and physiological timing, Ann. Rev. Plant Physiol. 27 (1976) 159-179.

[7] J.A. Kreps, S.A. Kay, Coordination of plant metabolism and development by the circadian clock, Plant Cell 9 (1997) 12351244.

[8] F. Nagy, S.A. Kay, N.H. Chua, A circadian clock regulates transcription of the wheat $c a b-1$ gene, Genes Dev. 2 (1988) $376-382$.

[9] H. Meyer, U. Thienel, B. Piechulla, Molecular characterization of the diurnal/circadian expression of the chlorophyll a/b-binding proteins in leaves of tomato and other dicotyledonous and monocotyledonous plant species, Planta 180 (1989) 5-15.

[10] M.L. Pilgrim, C.R. McClung, Differential involvement of the circadian clock in the expression of genes required for ribulose1,5-bisphosphate carboxylase/oxygenase synthesis, assembly and activation in Arabidopsis thaliana, Plant Physiol. 103 (1993) $553-564$.

[11] M.D. Deng, T. Moureaux, M.T. Leydecker, M. Caboche, Nitrate-reductase expression is under the control of a circadian rhythm and is light inducible in Nicotiana tabacum leaves, Planta 180 (1990) 257-261.

[12] A.N. Polidoros, J.G. Scandalios, Circadian expression of the maize catalase Cat 3 gene is highly conserved among diverse maize genotypes with structurally different promoters, Genetics 149 (1998) 405-415.

[13] B. Li, D.R. Geiger, W.J. Shieh, Evidence for circadian regulation of starch and sucrose synthesis in sugar beet leaves, Plant Physiol. 99 (1992) 1393-1399.

[14] J.C. Jang, J. Sheen, Sugar sensing in higher plants, Plant Cell 6 (1994) 1665-1679.

[15] P.A. Nakata, T.W. Okita, Differential regulation of ADP-glucose pyrophosphorylase in the sink and source tissues of potato, Plant Physiol. 108 (1995) 361-368.

[16] S. Takeda, S. Mano, M. Ohto, K. Nakamura, Inhibitors of protein phosphstsaes 1 and $2 \mathrm{~A}$ block the sugar-inducible gene expression in plants, Plant Physiol. 106 (1994) 567-574.

[17] R.G.F. Visser, A. Stolte, E. Jacobsen, Expression of a chimeric granule-bound starch synthase-GUS gene in transgenic potato plants, Plant Mol. Biol. 17 (1991) 691-699.

[18] S.N.I.M. Salehuzzaman, E. Jacobsen, R.G.F. Visser, Expression patterns of two starch biosynthetic genes in in vitro cultured cassava plants and their induction by sugars, Plant Sci. 98 (1994) $53-62$.

[19] J.C. Jang, P. León, L. Zhou, J. Sheen, Hexokinase as a sugar sensor in higher plants, Plant Cell 9 (1997) 5-19.
[20] F. Rook, N. Gerrits, A. Kortstee, M. Kampen, M. Borrias, P. Weisbeek, S. Smeekens, Sucrose-specific signalling represses translation of the Arabidopsis ATB2 bZIP transcription factor gene, Plant J. 15 (1998) 253-263.

[21] S. Smeekens, F. Rook, Sugar sensing and sugar-mediated signal transduction in plants, Plant Physiol. 115 (1997) 7-13.

[22] S. Lalonde, E. Boles, H. Hellmann, L. Barker, J.W. Patrick, W.B. Frommer, J.M. Ward, The dual function of sugar carriers: transport and sugar sensing, Plant Cell 11 (1999) 707-726.

[23] K.W. Yeh, R.H. Juang, J.C. Su, A rapid and efficient method for RNA isolation from plants with high carbohydrate content, Focus 13 (1991) 102-103.

[24] J. Sambrook, E.F. Fritsch, T. Maniatis, Molecular Cloning: A Laboratory Manual, 2nd ed., Cold Spring Harbor Laboratory, New York, 1989.

[25] S.N.I.M. Salehuzzaman, E. Jacobsen, R.G.F. Visser, Isolation and characterization of a cDNA encoding granule-bound starch synthase in cassava (Manihot esculenta Crantz) and its antisense expression in potato, Plant Mol. Biol. 23 (1993) 947-962.

[26] D.E. Somers, The physiology and molecular bases of the plant circadian clock, Plant Physiol. 121 (1999) 9-19.

[27] S.J. Wang, K.W. Yeh, H.L. Wang, J.C. Su, C.Y. Tsai, Light-induced circadian expression of starch granule-bound starch synthase gene in cell suspension cultures of sweet potato, Taiwania 44 (1999) 202-211.

[28] P.P. Dijkwel, P.A.M. Kock, R. Bezemer, P.J. Weisbeek, S.C.M. Smeekens, Sucrose represses the developmentally controlled transient activation of the plastocyanin gene in Arabidopsis thaliana seedlings, Plant Physiol. 110 (1996) 455-463.

[29] H.L. Wang, P.D. Lee, L.F. Liu, J.C. Su, Effect of sorbitol induced osmotic stress on the changes of carbohydrate and free amino acid pools in sweet potato cell suspension cultures, Bot. Bull. Acad. Sin. 40 (1999) 219-225.

[30] M. Ohto, K. Hayashi, M. Isobe, K. Nakamura, Involvement of $\mathrm{Ca}^{2+}$ signalling in the sugar-inducible expression of genes coding for sporamin and $\beta$-amylase of sweet potato, Plant J. 7 (1995) 297-307.

[31] P. Cohen, The structure and regulation of protein phosphatase, Annu. Rev. Biochem. 58 (1989) 453-508.

[32] G. Roblin, S. Sakr, J. Bonmort, S. Delrot, Regulation of a plant plasma membrane sucrose transporter by phosphorylation, FEBS Lett. 424 (1998) 165-168.

[33] Y.M. Li, J.E. Casida, Cantharidin-binding protein: Identification as protein phosphatase 2A, Proc. Natl. Acad. Sci. USA 89 (1992) 11867-11870.

[34] L.N. Sokolov, A. Dejardin, L.A. Kleczkowski, Sugars and light/ dark exposure trigger differenial regulation of ADP-glucose pyrophosphorylase genes in Arabidopsis thaliana (thale cress), Biochem. J. 336 (1998) 681-687. 